\title{
Clinical Features, Radiological Findings and Treatment Outcome in Patients with Pulmonary Nocardiosis - A Retrospective Analysis
}

\section{Prakrati Yadav}

All India Institutes of Medical Sciences Jodhpu

\section{Durga S Meena}

All India Institutes of Medical Sciences Jodhpu

Deepak Kumar ( $\nabla$ deepak1007sharma@gmail.com )

All India Institute of Medical Sciences Jodhpur https://orcid.org/0000-0001-6298-3565

\section{Gopal K Bohra}

All India Institutes of Medical Sciences Jodhpu

Vidhi Jain

All India Institutes of Medical Sciences Jodhpu

\section{Pawan Garg}

All India Institutes of Medical Sciences Jodhpu

Naveen Dutt

All India Institutes of Medical Sciences Jodhpu

\section{Ashwini Agarwal}

All India Institutes of Medical Sciences Jodhpu

\section{Mahendra K Garg}

All India Institutes of Medical Sciences Jodhpu

\section{Research}

Keywords: Pulmonary Nocardiosis, Cell mediate immunity, Modified ZN staining, Contrast-enhanced computer tomography, Trimethoprim sulfamethoxazole

Posted Date: April 23rd, 2021

DOI: https://doi.org/10.21203/rs.3.rs-442734/v1

License: (c) (1) This work is licensed under a Creative Commons Attribution 4.0 International License. Read Full License 


\section{Abstract}

Introduction - Lack of specific clinical features makes the diagnosis difficult in Pulmonary Nocardiosis. High index of suspicion is required for diagnosis especially in cohort pre-existing risk factors. This study aimed at describing the clinical and radiological characteristics and outcome in patients with Pulmonary Nocardiosis.

Methods - This was a retrospective observational study. Data of confirmed cases with Pulmonary Nocardiosis were collected from digital patient management system.

Results - Total eight cases of Pulmonary Nocardiosis were included. The mean age of patients was $50 \pm$ 14.3 years with female preponderance (62.5\%). The most common co-morbidity was chronic lung disease $(37.5 \%)$. The common clinical feature of pulmonary nocardiosis was cough with expectoration (50\%). The mean duration of symptoms was 18 days. The common radiological (CT thorax) finding were consolidation, bronchiectasis, mediastinal lymphdenoathy and nodularity (50\% each). One patient had extension of pulmonary disease in chest wall. Microbiological detection of Nocardia spp. was done in sputum samples (50\%) and in BAL samples (50\%). Culture was positive in two BAL samples. Intravenous empirical antibiotics in combination with oral trimethoprim sulfamethoxazole double standard $(15 \mathrm{mg} / \mathrm{kg}$ trimethoprim) was started at the time of diagnosis. Ceftriaxone and amikacin were commonly used antimicrobials.

Conclusion - Nocardia spp. commonly causes disease in patients with pre-exisitng lung pathological with or without defective cell mediated immunity. A high index of suspesion is required in patients with subacute to chronic respiratory symptoms, raised inflammatory markers and absence of common respiratory pathogens in evaluation.

\section{Introduction}

Pulmonary Nocardiosis is an infrequent diagnosis and common in patients with pre-existing lung condition with or without defective cell mediate immunity (1). Lack of specific clinical features and awareness in community physician further delays the diagnosis of Pulmonary Nocardiosis. Nocardia is an ubiquitous organism and due to abundence in environment the burden of Nocardiosis would be higher as compared to reported in lireture. Pulmonary Nocardiosis is not soughted as initial diagnosis and often missed. It has high mortality and early diagnosis of disease would be helpful in improving the survival (2). High index of suspicion is required for diagnosis especially in cohort with defective cell mediated immunity and other common organism have been ruled out. There is dearth of literature regarding Nocardiosis from developing countries. This study is reporting retrospective data of eight cases of pulmonary nocardiosis with focused on clinical features, associated lung conditions, risk factors, radiological findings and outcome of treatment.

\section{Method And Materials}


This was a retrospective observational study conducted in tertiary care center in western part of India. The study period was March 2019 to March 2020. Data of confirmed cases with Pulmonary Nocardiosis were collected from digital patient management system.

The diagnosis of Pulmonary Nocardiosis was considered when organism was isolated the respiratory samples (at least in two sputum samples and/or at least in one bronchoalveolar lavage) and negative for other microbial organism that could be responsible for the infection.

Collection of data included Data on demography, clinical symptoms, concurrent diseases, prior corticosteroid and/or immunosuppressive therapy, laboratory and radiological findings, dissemination to other organs and outcome of antibiotic therapy will be collected.

Microbiological identification of Nocardia Spp. was done under standard laboratory guidelines including either by direct microscopy and/or culture. Direct microscopy of sputum sample with modified ZielhNeelsen staining ( $1 \% \mathrm{H} 2 \mathrm{SO} 4$, Kinyoun method) showed filamentous, branching, and beaded acid-fast bacilli were considered as Nocardia Spp. Culture of sputum sample was incubated for 2-7 days at 35$36^{\circ} \mathrm{C}$ and identification of colonies was done on the basis of their phenotypic, metabolic, biochemical and growth tests on different temperatures. Interpretions of culture result were noted 24,48 and 72 hours after the incubation. Common pulmonary pathogens (bacterial, fungal, mycobacterium tuberculosis) were sought using Gram stain, Zielh-Neelsen stain and sputum culture, blood cultures.

Contrast-enhanced computer tomography (CECT) of the thorax was analyzed for all the patients. The various abnormality seen on CECT includes ground-glass opacity, consolidation, nodules, cavitatory lesion, bronchiectasis, mediastinal lymphadenopathy, presence and absence of pleural and pericardial effusion were also noted.

Data were summarized and tabulated, continues variables showed in the form of mean \pm standard deviation and categorical variables in the form of percentage and frequencies.

\section{Results}

This study included eight cases of Pulmonary Nocardiosis, which was diagnosed in a period of one year from March 2019 to March 2020. The diagnosis of pulmonary nocardiosis was made in patient who presented with respiratory symptoms and either positive for modified acid-fast staining in sputum or positive culture for Nocardia spp. in sputum sample.

Demographic characteristic and underlying co-morbidities are showed in Table 1. The mean age of patients was $50 \pm 14.3$ years (range $29-72$ years) with female preponderance (62.5\%). All patients have at least one underlying comorbidities as depicted in Table 1 . The most common co-morbidity was chronic lung disease found in 3 (37.5\%) patients ( 2 had chronic obstructive pulmonary disease and one had posttubercular lung fibrosis). 
Table 1

Demography and underlying risk factors of patients with Pulmonary Nocardiosis

\begin{tabular}{|ll|}
\hline Characteristic & $\mathbf{n}(\%)$ \\
\hline Total Patients & 8 \\
\hline Mean age (years) & $50 \pm 14.3$ \\
\hline Sex distribution & \\
\hline Male & $3(37.5 \%)$ \\
\hline Female & $5(62.5 \%)$ \\
\hline Underlying conditions & \\
\hline Chronic lung disease & $3(37.5 \%)$ \\
\hline Chronic kidney disease & $1(12.5 \%)$ \\
\hline Malignancy & $1(12.5 \%)$ \\
\hline Steroid and Immunosuppressive drugs (Autoimmune disease) & $1(12.5 \%)$ \\
\hline Pancytopenia & $1(12.5 \%)$ \\
\hline Chronic kidney disease & $1(12.5 \%)$ \\
\hline Idiopathic CD4 lymphocytopenia & $1(12.5 \%)$ \\
\hline
\end{tabular}

The commonest clinical feature of pulmonary nocardiosis was cough with expectoration, which was present in all patients and fever was present only in $4(50 \%)$ patients (Table 2). The mean duration of symptoms was 18 days (range, 7-30) and maximum patients had symptoms for more than 14 days. Two patients were clinically diagnosed as pulmonary tuberculosis and was on anti-tubercular therapy. One patient had past history of pulmonary tuberculosis 3 year back and received complete 6 months' therapy. Anemia (10.67 \pm 1.87 in gm\%) and leukocytosis (10182 \pm 5842 cell/cumm) were present in $42.85 \%$ of patients and inflammatory markers were significantly raised in most of patients (HsCRP $84.5 \pm$ 73.11 and ESR $53.14 \pm 14.67$ ) (Table 2). One patient had associated idiopathic CD4 T cell lymphocytopnia. Absolute CD 4 count of this patient was $212 \mathrm{cell} / \mu \mathrm{L}$ and $191 \mathrm{cell} / \mu \mathrm{L}$ on two occasion 3 months apart and negative for HIV-1 and HIV-2 on both occasion. 
Table 2

Clinical and laboratory features of the patients with Pulmonary Nocardiosis

\begin{tabular}{|ll|}
\hline Characteristic & $\mathbf{n}(\%)($ Mean \pm SD) \\
\hline Clinical Features & \\
Fever & $4(50 \%)$ \\
Cough & $8(100 \%)$ \\
\hline Expectoration & $8(100 \%)$ \\
\hline Breathlessness & $5(62.5 \%)$ \\
\hline Weight loss & $3(37.5 \%)$ \\
\hline Laboratory Investigations & \\
\hline Anemia & $3(37.5 \%)$ \\
\hline Leukocytosis & $3(37.5 \%)$ \\
\hline Neutrophilia & $1(12.5 \%)$ \\
\hline lymphocytosis & $1(12.5 \%)$ \\
\hline High HsCRP & $7(87.5 \%)$ \\
\hline High ESR & $8(100 \%)$ \\
\hline
\end{tabular}

The radiological (CT thorax) finding were showed in Table 3. Consolidation, bronchiectasis, mediastinal lymphdenoathy and nodularity were common finding (Fig. 1). Involvement of unilateral lung parenchyma was found in $4(50 \%)$ patients. One patient had invasive disease with extension in chest wall (Table 3 ). 
Table 3

Finding in computer tomography (CT) of thorax

in patients with Pulmonary Nocardiosis

\begin{tabular}{|ll|}
\hline CT Thorax finding & $\mathbf{n}(\%)$ \\
\hline Consolidation & $3(50 \%)$ \\
\hline Ground Glass Opacity & $2(25 \%)$ \\
\hline Cavitatory lesion & $3(37.5 \%)$ \\
\hline Nodules & $4(50 \%)$ \\
\hline Bronchiectasis & $4(50 \%)$ \\
\hline Fibrotic changes & $4(50 \%)$ \\
\hline Mediastinal Lymphadenoapthy & $4(50 \%)$ \\
\hline Pleural effusion & $1(12.5 \%)$ \\
\hline Pericardial effusion & $1(12.5 \%)$ \\
\hline
\end{tabular}

Microbiological detection of Nocardia spp. was done in two consecutive sputum samples in four patients $(50 \%)$ and in BAL samples in four patients (50\%). Culture was positive in two BAL samples.

Intravenous empirical antibiotics in combination with oral trimethoprim sulfamethoxazole double standard (15 mg/kg trimethoprim) was started at the time of diagnosis. Ceftriaxone and amikacin were commonly used antimicrobials (Table 4). One patient (62-year-old male) died during treatment, who had underlying squamous cell carcinoma of buccal mucosa with brain metastasis.

Table 4

Antibiotic regimens and outcome in patients with Pulmonary Nocardiosis

\begin{tabular}{|ll|}
\hline Antibiotic combinations & Treated patients/deaths \\
\hline Ceftriaxone + amikacin + trimethoprim sulfamethoxazole DS & $4 / 0$ \\
\hline Imipenem + amikacin + linezolid & $1 / 1$ \\
\hline Amikacin + trimethoprim sulfamethoxazole DS & $3 / 0$ \\
\hline
\end{tabular}

\section{Discussion}

Nocardia is an aerobic, gram-positive filamentous bacteria and commonly affects respiratory system in human. Nocardia can cause disseminated disease involving central nervous system (CNS), and skin or rarely other organ (3). It can cause acute, subacute as well as chronic disease and high suspicion of index is required for diagnosis. Pulmonary Nocardiosis is consider to be an opportunistic infection and occurred mainly in patients with defective cellular immunity. Due to lack of specific clinical features, diagnosis of pulmonary nocardiosis turn out to be difficult. In most of the time pulmonary tuberculosis, 
lung abscess, bronchiectasis with secondary bacterial infection, invasive fungal infection etc. are keeping as initial diagnosis in patients with Pulmonary Nocardiosis. This study is focused on clinical and laboratory features in patients with pulmonary nocardiosis to increase awareness among community physician.

The mean age of patients in this study was $50 \pm 14.3$ years and disease was common in female. Contrary to this, previously literature had higher incidence of pulmonary nocardiosis in male $(1,4,5,6,7)$. This difference may be due to smaller number of cases or lack of larger studies. Chronic obstructive lung disease (COPD) was the most common risk factor associated in around 40\% of cases. COPD and chronic steroid use are the important pre-existing factor for Pulmonary Nocardiosis and more than half of patients had above comorbidities in previous studies $(1,2,3,8,9)$. Patients with malignancy, leukemia, HIV, diabetes, on cytotoxic chemotherapy and organ transplant recipient are also have increased risk for Nocardiosis. Association of Idiopathic CD4 lymphocytopenia and Pulmonary Nocardiosis is less studied. In this study one patients had low CD4 (less than 300 cell/ $\mu \mathrm{L}$ ) on two occasion with negative for HIV-1 and 2. We recommend that CD 4 count should be done in every case of Nocardiosis to find association with ICL and further prophylaxis.

The duration of disease was subacute or chronic in most of cases but one patient was presented with 6 days of illness, who also had pancytopenia. High suspicion should be kept even in acute presentation especially in patients with risk factors (3). The common presenting clinical features were cough, expectoration and breathlessness while fever was seen only in half of cases. These clinical features are non-specific and often directed towards tuberculosis or fungal infection. Invasive nature of disease is important diagnostic clue and one patient had extension of disease in chest wall, which was diagnosed in CECT thorax. Though there were no specific abnormalities seen in hematological profile. Anemia, lymphocytosis, neutrophilia and raised inflammatory markers were found similar to other cases series (1, 10). Persistent symptoms, raised inflammatory markers and lack of other infective etiology in patient with chronic lung disease become a high index of suspicion for Pulmonary Nocardiosis.

Common CT thorax findings in Pulmonary Nocardiosis were parenchymal nodules, consolidation, mediastinal lymphadenopathy with or without cavitation present in around $70 \%$ of patients. Above CT abnormality in immunocompromised patients with high clinical suspicion may suggest the likelihood of Pulmonary Nocardiosis (11). Confirmation of diagnosis is requiring identification and isolation of Nocardia spp. In modified acid-fast staining using $1 \%$ sulfuric acid and/or culture in sputum or BAL sample (1). In this study all patients had positive for modified acid-fast staining, showed filamentous branching bacilli consisted with Nocardia spp. BAL was done in four cases and out of them 2 had positive culture for Nocardia spp. Species identification was not done due to resource constrain. A clinical background should be taken care during isolation of Nocardia spp. to avoid over diagnosis and superfluous use of antibiotic.

These is no definitive treatment guideline for nocardiosis and most of the data are from retrospective studies. Combination antibiotic therapy with Trimethoprim-sulfomethoxazole is, in a dose of 25-50 
$\mathrm{mg} / \mathrm{kg}$ per day of sulfamethoxazole is the most widely used in treatment $(1,3)$. Amikacin, ceftriaxone, imipenem and linezolid have been showed effectiveness in various combination. Pulmonary disease with moderate symptoms ( $50 \%$ cases) were treated with combination of ceftriaxone plus amikacin plus Trimethoprim-sulfomethoxazole showed effective cure in this study. Patients should be treated for at least 6 months' duration or till resolution of disease especially in immunocompromised patients (12). Mortality is high in nocardiosis and one long term study showed $41 \%, 64 \%$ and $100 \%$ mortality in pulmonary, disseminated and CNS disease respectively (2). Six patients were treated for 6 months, one patient treated for 12 months and one died during tretament in this study.

\section{Conclusion}

Pulmonary Nocardiosis doesn't have any pathogonomic clinical featutes and radiological findings. Nocardia spp. commonly causes disease in patients with pre-exisitng lung pathological with or without defective cell mediated immunity. A high index of suspesion is required in patients with subacute to chronic respiratory symptoms, raised inflammatory markers and absence of common respiratory pathogens in evaluation.

\section{Abbreviations}

CECT - Contrast-enhanced computer tomography

HsCRP - High Sensitive C reactive protein

ESR - Erthrocyte Sedimentation Rate

CD4 - Culster Diffentiation 4

BAL - Bronchoalveolar Lavage

CNS - Central Nervous Sysytem

COPD - Chronic Obstructive Pulmonary Disease

HIV - Human Immunodeficiency Syndrome

ICL - Idiopathic CD4 Lymphocytopenia

\section{Declarations}

Ethics approval and consent to participate- Permission of the study has been taken from Institutional Ethical committee (AlIMS/JDH/2021/3268).

Funding- This research did not receive any specific grant from funding agencies in the public, commercial, or not-for-profit sectors. 
Availability of Data and Materials - The datasets used and/or analysed during the current study available from the corresponding author on reasonable request.

Consent for publication - The patient has given written consent for his personal and clinical details. The authors certify that they have obtained all appropriate patient consent forms. In the form, the patients have given their consent for their images and other clinical information to be reported in the journal. The patients understand that their names and initials will not be published and due efforts will be made to conceal their identity.

\section{Competing interests - Nil}

Authors' contributions -: PY : Investigation, Resources, Writing- Original draft preparation. DSM: Investigation, Resources, Writing- Original draft preparation. DK : Methodology, Data Curation, Writing Original Draft. GKB : Conceptualization, Methodology, Data Curation, Writing - Review \& Editing, Supervision. VJ: Methodology, Investigation, Resources, Writing - Review \& Editing. PG : Conceptualization, Methodology, Data Curation, Writing- Original draft preparation. ND : Investigation, Resources, Writing - Review \& Editing. AA : Conceptualization, Methodology, Writing- Original draft preparation. MKG : Conceptualization, Methodology, Writing - Review \& Editing, Supervision.

'All authors have read and approved the manuscript'.

\section{Acknowledgement - 'Not Applicable'}

\section{References}

1. Singh A, Chhina D, Soni RK, Kakkar C, Sidhu US. Clinical spectrum and outcome of pulmonary nocardiosis: 5-year experience. Lung India. 2016;33:398-403.

2. Martínez Tomás R, Menéndez Villanueva R, Reyes Calzada S, Santos Durantez M, Vallés Tarazona $\mathrm{JM}$, Modesto Alapont M, et al. Pulmonary nocardiosis: risk factors and outcomes. Respirology. 2007;12(3):394-400.

3. Aggarwal D, Garg K, Chander J, Saini V, Janmeja AK. Pulmonary nocardiosis revisited: A case series. Lung India. 2015;32(2):165-8.

4. Garcia-Bellmunt L, Sibila O, Solanes I, Sanchez-Reus F, Plaza V. Pulmonary nocardiosis in patients with COPD: Characteristics and prognostic factors. Arch Bronconeumol. 2012;48:280-5.

5. Mootsikapun P, Intarapoka B, Liawnoraset W. Nocardiosis in Srinagarind Hospital, Thailand: Review of 70 cases from 1996-2001. Int J Infect Dis. 2005;9:154-8.

6. Castro JG, Espinoza L. Nocardia species infections in a large county hospital in Miami: 6 years experience. J Infect. 2007;54:358-61.

7. Matulionyte R, Rohner P, Uçkay I, Lew D, Garbino J. Secular trends of Nocardia infection over 15 years in a tertiary care hospital. J Clin Pathol. 2004;57:807-12. 
8. MartínezTomás R, Menéndez Villanueva R, Reyes Calzada S, Santos Durantez M, VallésTarazona $\mathrm{JM}$, Modesto Alapont M, et al. Pulmonary nocardiosis: Risk factors and outcomes. Respirology. 2007;12:394-400.

9. Muñoz J, Mirelis B, Aragón LM, Gutiérrez N, Sánchez F, Español M, et al. Clinical and microbiological features of nocardiosis 1997-2003. J Med Microbiol. 2007;56:545-50.

10. Yang M, Xu M, Wei W, Gao H, Zhang X, Zhao H, et al. Clinical findings of 40 patients with nocardiosis: A retrospective analysis in a tertiary hospital. Exp Ther Med. 2014;8(1):25-30.

11. Liu B, Zhang Y, Gong J, Jiang S, Huang Y, Wang L, et al. CT findings of pulmonary nocardiosis: a report of 9 cases. J Thorac Dis. 2017;9(11):4785-90.

12. Wilson JW. Nocardiosis: Updates and clinical overview. Mayo Clin Proc. 2012; 87:403-7.

\section{Figures}

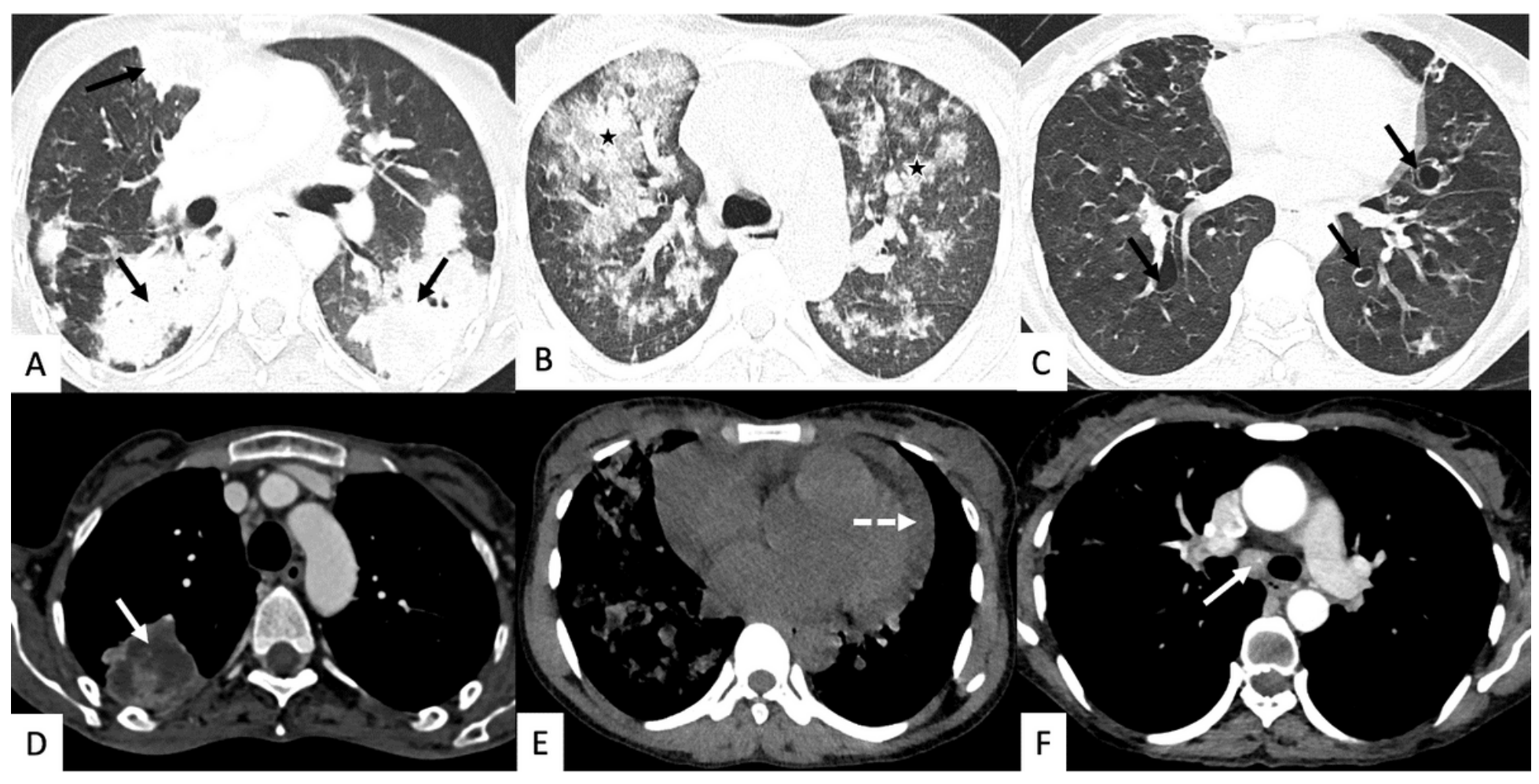

Figure 1

Computed tomography (CT) thorax in different patients. Case 1: high resolution CT (HRCT) (A) and contrast enhanced CT (CECT) (D) showing multifocal patchy area of consolidation (black arrows) with internal necrosis (white arrow). Case 2: HRCT (B) and non contrast CT (NCCT) (E) showing diffuse consolidation and ground glass in bilateral lungs (asterisk) with pericardial effusion (dashed arrow). Case 3: HRCT (C) and CECT (F) showing bronchiectasis (black arrows) with multiple parenchymal nodules and mediastinal lymphadenopathy (white arrow). 